\title{
DISTRIBUTIONS ISOTOPIQUES DES PRODUITS DE TRANSFERTS TRẼS INÉLASTIQUES ENTRE IONS LOURDS
}

\author{
J. C. JACMART, N. FRASCARIA, N. POFFÉ, P. COLOMBANI, H. DOUBRE, \\ M. RIOU, J. C. ROYNETTE et C. STÉPHAN \\ Institut de Physique Nucléaire, B.P. 1, 91406 Orsay, France
}

\begin{abstract}
Résumé.- On compare les distributions isotopiques expérimentales des produits de transfert très inélastiques obtenus par les réactions ${ }^{40} \mathrm{Ca}(284 \mathrm{MeV})+{ }^{40} \mathrm{Ca}$ et ${ }^{40} \mathrm{Ar}(295 \mathrm{MeV})+{ }^{232} \mathrm{Th}$.

Abstract. - Experimental isotopic distributions of deep inelastic transfer products are compared for the two systems ${ }^{40} \mathrm{Ca}(284 \mathrm{MeV})+{ }^{40} \mathrm{Ca}$ and ${ }^{40} \mathrm{Ar}(295 \mathrm{MeV})+{ }^{232} \mathrm{Th}$. The first case, for which primary products should be symmetric around ${ }_{20}^{40} \mathrm{Ca}$, shows a shift of about $\Delta Z=-2$ and $\Delta N=-1.5$ due to evaporation, and a strong 2 protons +2 neutrons structure. The second case shows a wider distribution probably due to more partial equilibrium.
\end{abstract}

On sait que l'interaction entre ions lourds au-dessus de la barrière coulombienne donne lieu, avec une fraction importante de la section efficace totale, à des réactions de transferts à deux corps principaux dans la voie de sortie. Deux phénomènes distincts sont observés pour ces réactions :

i) Les transferts quasi élastiques, phénomènes directs observés au voisinage de l'angle d'effleurement avec peu de perte d'énergie.

ii) Les transferts très inélastiques (deep inelastic) caractérisés par un nombre important de nucléons échangés, une perte très importante d'énergie cinétique et une distribution angulaire étalée. Ce phénomène est généralement interprété comme correspondant à un recouvrement important des deux noyaux, avec un paramètre d'impact inférieur à la distance d'effleurement. L'interaction nucléaire comprenant un terme de friction agit à la fois, sur la direction des trajectoires de sortie qu'elle ramène vers l'avant (ou même vers des angles négatifs) par rapport à une trajectoire coulombienne non perturbée, et sur l'énergie cinétique de sortie qu'elle diminue.

Expérimentalement, ces deux phénomènes sont assez bien séparés suivant les cas, soit par l'angle d'observation, soit par l'énergie cinétique de sortie. $\mathrm{La}$ répartition isotopique des produits de transfert observés dépend principalement de trois facteurs :

1) les rapports $N / Z$ du projectile et de la cible,

2) le degré d'équilibre partiel entre cible et projectile, lié au temps pendant lequel les noyaux restent en interaction et qui peut être de l'ordre de grandeur de la période de rotation du système composite,

3) l'énergie d'excitation qui conduit à une évaporation de particules légères avant détection.
Nous montrons ci-dessous, sous forme de cartes, les distributions isotopiques des produits légers, observés dans deux cas très différents :

$$
\begin{array}{lll}
{ }^{40} \mathrm{Ca}+{ }^{40} \mathrm{Ca} & E_{\text {Lab }}=284 \mathrm{MeV} & \theta_{\text {Lab }}=10^{\circ} \\
{ }^{40} \mathrm{Ar}+{ }^{232} \mathrm{Th} & E_{\text {Lab }}=295 \mathrm{MeV} & \theta_{\text {Lab }}=18^{\circ} .
\end{array}
$$

Dans le premier cas [1], l'angle d'observation est voisin de l'angle d'effleurement $\left(14^{\circ} \mathrm{lab}\right)$ et les deux phénomènes sont observés; mais l'énergie incidente étant très supérieure à la barrière coulombienne $\left(B_{\mathrm{lab}}=110 \mathrm{MeV}\right)$, l'énergie cinétique des produits détectés permet de séparer les deux régions très inélastique et quasi élastique : les produits de transferts très inélastiques ont une énergie cinétique voisine de la répulsion coulombienne, ce qui correspond en moyenne à une énergie d'excitation totale de 70 à $80 \mathrm{MeV}$, répartie à peu près également entre les deux produits de réaction. La séparation entre les deux phénomènes correspond à une énergie d'excitation de l'ordre de $30 \mathrm{MeV}$.

Du fait de la symétrie de la voie d'entrée de cette réaction, les processus de stripping et de pick-up sont indiscernables. Dans l'hypothèse d'un processus primaire à deux corps, non suivi d'évaporation, les sections efficaces intégrées à un angle donné devraient donc être égales pour deux noyaux symétriques par rapport à ${ }^{40} \mathrm{Ca}$. Tout écart par rapport à cette symétrie doit donc être interprété comme provenant de l'émission de particules légères, pendant ou après l'interaction.

Dans le cas ${ }^{40} \mathrm{Ar}+{ }^{232} \mathrm{Th}$ [2], l'angle d'effleurement est à $47^{\circ}(\mathrm{lab})$ et seuls apparaissent à $18^{\circ}$ les produits de transfert très inélastiques dont l'énergie cinétique moyenne est égale à $80 \%$ de l'énergie de répulsion coulombienne (cas sphérique). Ceci cor- 
respond à une énergie d'excitation de l'ordre de $100 \mathrm{MeV}$ qui serait principalement emportée par le noyau lourd de recul dont la densité de niveaux est beaucoup plus élevée que celle du noyau léger détecté.

La figure 1 représente la distribution isotopique des produits de transfert quasi élastique dans l'interaction $\left({ }^{40} \mathrm{Ca}+{ }^{40} \mathrm{Ca}\right)$. Les courbes d'égale section efficace (CM) s'organisent en une figure symétrique dont le centre est décalé par rapport au point $N=Z=20$ de $\Delta Z=-0,25$ et dont l'axe a subi une légère rotation vers la ligne de stabilité. Ceci peut provenir d'une évaporation peu importante compatible avec une énergie d'excitation inférieure à $30 \mathrm{MeV}$. On remarque un renforcement net de la section efficace de ${ }_{18}^{36} \mathrm{Ar}$ qu'on ne retrouve pas pour le noyau symétrique ${ }_{22}^{44} \mathrm{Ti}$.

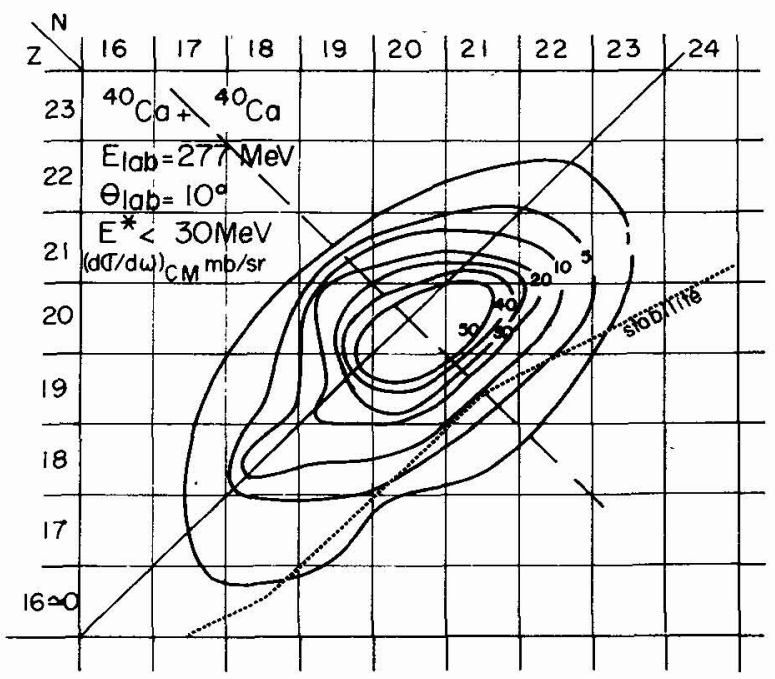

Fig. 1. - Distribution des sections efficaces des produits de transfert quasi élastique dans l'interaction ${ }^{40} \mathrm{Ca}+{ }^{40} \mathrm{Ca}$.

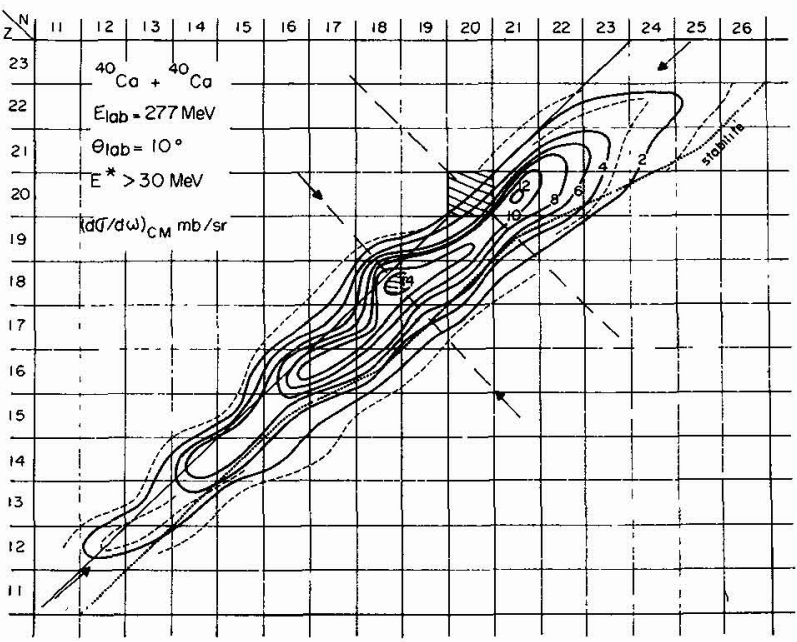

FIG. 2. - Distribution des sections efficaces des produits de transfert très inélastique dans l'interaction ${ }^{40} \mathrm{Ca}+{ }^{40} \mathrm{Ca}$. Les axes de symétrie sont indiqués par des flèches.
La figure 2 représente la distribution isotopique pour les produits de transfert très inélastique de la réaction $\left({ }^{40} \mathrm{Ca}+{ }^{40} \mathrm{Ca}\right)$. On observe une distribution également symétrique, beaucoup plus allongée, dont le sommet est décalé de $\Delta Z=-2$ et $\Delta N=-1,5$ par rapport au point $N=Z=20$. Pour ces noyaux excédentaires en protons, les énergies de liaison sont plus élevées pour les neutrons que pour les protons. Il est donc probable que les noyaux formés avec une énergie d'excitation de 35 à $40 \mathrm{MeV}$ et un moment angulaire non négligeable puissent évaporer des particules $\alpha$ et des protons. Il apparaît d'autre part un renforcement remarquable des sections efficaces de formation des noyaux éloignés d'un nombre entier de particules $\alpha$ à partir du centre de symétrie de la distribution.

Il est intéressant de comparer cette distribution à celle obtenue pour la réaction ${ }^{40} \mathrm{Ar}+{ }^{232} \mathrm{Th}$ (Fig. 3). Dans ce cas, les produits de réaction légers, riches en neutrons comme le projectile et la cible, sont formés avec une énergie d'excitation faible [2]. On s'attend à une évaporation préférentielle d'un petit nombre de neutrons. On observe aussi une distribution symétrique des sections efficaces dont le maximum est situé au voisinage du projectile, avec un léger déplacement positif en $N$ (lié sans doute au grand excès de neutrons dans la cible). La distribution est nettement plus étendue que la précédente, les largeurs suivant les axes de symétrie étant toutes deux supérieures de $30 \%$ à celles observées dans le cas du Ca.

Il apparait, par ailleurs, un renforcement des sections efficaces avec la même périodicité $\langle\alpha »$ que précédemment : dans la mesure où l'évaporation $\alpha$ serait négligeable dans le cas $\mathrm{Ar}+\mathrm{Th}$, cet effet devrait être attribué au processus primaire et relié notamment aux fluctuations corrélées des bilans en énergie et des énergies d'excitation des produits primaires.

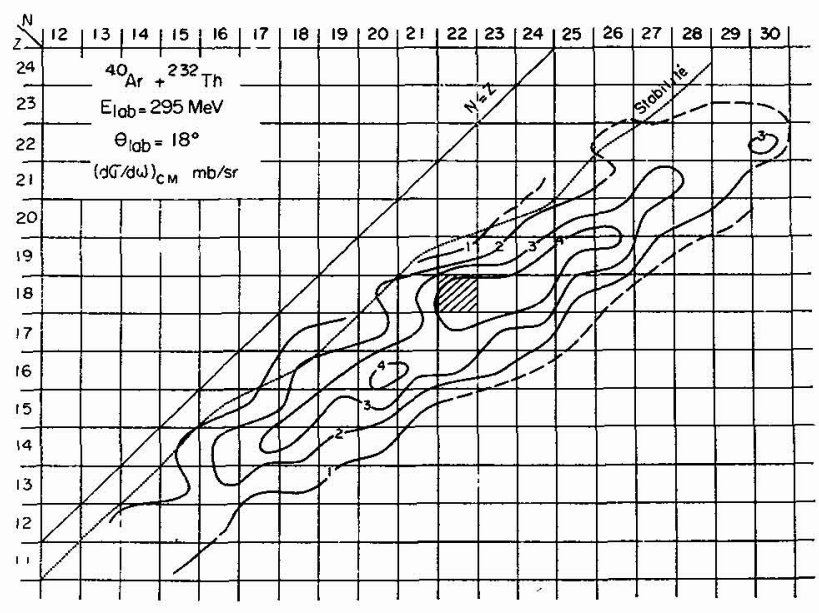

Fig. 3. - Distribution des sections efficaces des produits légers de transfert très inélastique dans l'interaction ${ }^{40} \mathrm{Ar}+{ }^{232} \mathrm{Th}$. 
Un calcul statistique d'évaporation devrait confirmer ces conclusions et permettrait de proposer dans les deux cas les distributions isotopiques du processus primaire. La forme de ces distributions traduit le degré d'équilibre partiel atteint entre projectile et cible au cours de l'interaction. Associées aux distributions angulaires et énergétiques, les distributions isotopiques doivent donc permettre d'obtenir des renseignements sur les effets de friction ou de viscosité dans la matière nucléaire.

Note ajoutée aux épreuves. - Contrairement à la valeur indiquée sur les figures 1 et $2(277 \mathrm{MeV})$, l'énergie moyenne des réactions ${ }^{40} \mathrm{Ca}+{ }^{40} \mathrm{Ca}$ est $284 \mathrm{MeV}$.

\section{Bibliographie}

[1] Colombani, P. et al., Phys. Lett. 55B (1975) 45.

[2] JacmarT, J. C. et al., Nucl. Phys. A 242 (1975) 175. 